\title{
Robust Output Regulation of Switched Multi-agent Systems
}

\author{
ZHANG Xiaoqi \\ Liaoning construction vocational college, Liaoyang 111000, P. R. China \\ E-mail: eyes8888@126.com
}

\begin{abstract}
Keywords: switched multi-agent systems, robust output regulation, distributed control.
\end{abstract}
\begin{abstract}
The robust output regulation problem of switched linear multi-agent systems is studied in this paper. The dynamics of each agent are described by a switched linear system. According to the characteristics of agent network and switched systems, we put forward an agent-dependent average dwell time method. Owing to the limited information exchange between agents, distributed dynamic controllers are designed. Finally, simulation results show the effectiveness of the proposed solutions.
\end{abstract}

\section{Introduction}

Switched systems, as a hot research field, is composed of a family of continuous-time or discretetime subsystems and a rule determining the switching between the subsystems [1-3]. Stability, as a key issue in the study of switched systems, has attracted much attention. Several effective approaches have been developed, among which the average dwell time method is one of the most effective methods. The average dwell time method is firstly presented in [4], the switching is slowon-the-average among stable subsystems. In the case of linear switched systems consisting of both Hurwitz stable and unstable subsystems in [5], if the average dwell time is chosen sufficiently large and the total activation time of unstable subsystems is relatively small compared with that of Hurwitz stable subsystems, the exponential stability of a desired degree is guaranteed.

On the other hand, output regulation, as one of the most fundamental problems, its purpose is to design a controller to ensure the tracking of a class of a reference signals and rejection of a class of disturbance while maintaining the internal stability of the closed-loop system. For the multi-agent systems, output regulation is also one of the fundamental problems. If each agent can obtain the information of the exosystem, the output regulation problem can be easily handled by decentralized control [6]. In fact, this is not often possible. Thus, cooperative control of multi-agent systems Conform to the actual situation and becomes the focus of the study. For the no-switched multi-agent systems, by a dynamic full information distributed control scheme in [7] and devising distributed observer network in [8] respectively, the output regulation problem can be solved. In [9], the cooperative robust output regulation problem is studied by both distributed state feedback and distributed output feedback control. Based on internal model principle, distributed control scheme was adopted to achieve the robust regulated objective in [10]. For the switched multi-agent systems, in the existing literatures, the switching of multi-agent systems includes two kinds of switching. One is switching of interconnections of agents, and the other is that each agent is a switched system and the switching takes places between subsystems of an agent. Most results of the output regulation problem of switched multi-agent systems are for the former one, namely, the switching between interconnections of agents [11-13]. However, few results about output regulation problem of multi-agent systems whose agents are switched systems have appeared [14].

In this paper we study the robust output regulation problem of multi-agent systems in which each agent dynamics are represented by a switched linear system. Due to the existing of the uncertain, we will study coperative semi-global robust output regulation problem for the class of uncertain switched linear multi-agent systems. Owing to not every agent can obtain the information of the exosystem, a distributed dynamic controller of synchronous switching with the agents is set up. Base on the characteristics of multiagent systems and the linear switched systems, we present a new method, called an agent-dependent average dwell time method. Compared with the existing 
literature [14], the switched linear multi-agent systems considered have more general form on structure and a new agent-dependent average dwell time method is proposed.

The remainder of the paper is organized as follows. The preliminaries and problem statements are discussed in Section 2. In Section 3, the main results are given. An example is given in Section 4. Section 5 draws a conclusion.

Notation: The information exchange among agents is denoted by the directed graph $G=(\vartheta, \varepsilon) . \vartheta$ $=\{1,2, \cdots, N\}$ is the set of node and $\varepsilon \in \vartheta \times \vartheta$ is the set of edges. $A=\left[a_{i j}\right], i, j=1,2, \cdots, N$ is a adjacency matrix of the graph $G$. The neighbor set of the $i$-th agent is denoted by $\Phi_{i}=\{j:(j, i) \in \varepsilon, i$ $=1,2, \cdots, N\}$. Let a diagonal matrix $D=\operatorname{diag}\left\{\sum_{j=1}^{N} a_{1 j}, \sum_{j=1}^{N} a_{2 j}, \cdots, \sum_{j=1}^{N} a_{N j}\right\}$, the Laplacian matrix $L$ will be defined $L=D-A$. Let $a_{i 0}$ describe the connection weight from the $i$-th agent to the leader, and $a_{i 0}>0$ if the $i$-th agent can obtain information from the leader, otherwise $a_{i 0}=0$. Set a diagonal matrix $A_{0}=\operatorname{diag}\left\{a_{10}, a_{20}, \cdots, a_{N 0}\right\}$. Let $H=L+A_{0}$. We know $H$ is positive definite if and only if node 0 is globally reachable [15].

\section{Problem statement}

In this paper, we consider the following switched linear multi-agent systems. The dynamics of agents are described as

$$
\begin{gathered}
\dot{x}_{i}{ }_{i}=A_{i \sigma_{i}(t)}(\omega) x_{i}+B_{i \sigma_{i}(t)}(\omega) u_{i}+E_{i \sigma_{i}(t)}(\omega) v, \\
\mathrm{y}_{i}=C_{i}(\omega) x_{i}, i=1,2, \cdots, N,
\end{gathered}
$$

where $x_{i} \in R^{n_{i}}, u_{i} \in R^{m_{i}}, y_{i} \in R^{q}$ are the state, control input and output of the $i$-th agent respectively. $\sigma_{i}(t): \rightarrow M_{i}=\left\{1,2, \cdots, \rho_{i}\right\}$ is a piecewise constant function of time $t$ called a switching signal of the $i$ th agent.

$$
\begin{aligned}
& A_{i j_{i}}(\omega)=A_{i j_{i}}+\Delta A_{i j_{i}}, B_{i j_{i}}(\omega)=B_{i j_{i}}+\Delta B_{i j_{i}}, \\
& E_{i j_{i}}(\omega)=E_{i j_{i}}+\Delta E_{i j_{i}}, C_{i}(\omega)=C_{i}+\Delta C_{i}, i=1,2, \cdots, N, j_{i} \in M_{i} .
\end{aligned}
$$

The matrices $A_{i j_{i}}, B_{i j_{i}}, E_{i j_{i}}$ and $C_{i}$ represent the nominal part of the plant while $\Delta A_{i j_{i}}$, and so forth represent the uncertain part. $v \in R^{q}$ is the exogenous signal which represents the reference input to be tracked and (or) the disturbance to be rejected. It is assumed to be generated by a so-called exosystem defined by

$$
v^{\cdot}=S v, \quad y_{0}=-F v,
$$

where $y_{0}$ is the reference output and $S$ is a constant matrix.

We can define the regulated error output for the $i$-th agent as follows:

$$
e_{i}=y_{i}-y_{0}=C_{i}(\omega) x_{i}+F v, i=1,2, \cdots, N \text {. }
$$

We consider the following distributed dynamic control law

$$
\begin{gathered}
u_{i}=K_{i \sigma_{i}(t)} x_{i}+G_{i \sigma_{i}(t)} \xi_{i}, \\
\xi_{i}=S \xi_{i}+\alpha\left[\sum_{j \in \Phi_{i}} a_{i j}\left(\xi_{j}-\xi_{i}\right)+a_{i 0}\left(v-\xi_{i}\right)\right],
\end{gathered}
$$

where $K_{i \sigma_{i}(t)} \in R^{n_{i} \times m_{i}}, G_{i \sigma_{i}(t)} \in R^{m_{i} \times q}$ are gain matrices to be determined later and $a_{i j}, a_{i 0} \in H, i=$ $1,2, \cdots, N, j_{i} \in M_{i}$. $j$ belongs to the neighbor set of the $i$-th agent.

Then, the output regulation problem of the switched linear multi-agent system (1) is stated below. The output regulation problem: Given the systems (1) and the exosystem (2), find the control law (4) and switching signals $\sigma_{i}(t)$ such that

( i ) When $\omega=0$ and $v=0$, the closed-loop system is asymptotically stable;

( ii ) There exists an open neighborhood $\Omega$ of $\omega=0$ and for any initial condition $x_{i}(0), \eta_{i}(0), i$ $=1,2, \cdots, N$ and $v(0)$, the tracking error satisfies

$$
\lim _{t \rightarrow \infty} e_{i}(t)=0 .
$$

The solvability of the output regulation depends on some standard assumptions listed below. 
Assumption 1: The eigenvalues of the matrix $S$ have no negative real part.

Assumption 2: For $i=1,2, \cdots, N, j_{i}=1,2, \cdots, \rho_{i}$, the linear matrix equations

$$
\begin{aligned}
& X_{i}(\omega) S=A_{i j_{j}}(\omega) X_{i}(\omega)+B_{i j_{i}}(\omega) U_{i j_{i}}(\omega)+E_{i j_{i}}(\omega), \\
& 0=C_{i}(\omega) X_{i}(\omega)+F,
\end{aligned}
$$

have the solution pairs $\left(X_{i}(\omega), U_{i j_{i}}(\omega)\right)$ respectively.

Assumption 3: Matrix pairs $\left(A_{i j_{i}}, B_{i j_{j}}\right), i=1,2, \cdots, N, j_{i} \in M_{i}$ are stabilizable.

Assumption 4: The node 0 (the exosystem) is globally reachable.

In order to solve the output regulation problem, we need the following lemma.

Lemma 1: Consider the switched uncertain linear system:

$$
\begin{gathered}
\dot{x}_{c}=A_{c \sigma_{c}(t)}(\omega) x_{c}+B_{c \sigma_{c}(t)}(\omega) v, \\
v^{\cdot}=\Gamma v, \\
e=C_{c}(\omega) x_{c}+D_{c} v,
\end{gathered}
$$

where $x_{c} \in R^{n_{c}}, v \in R^{q_{c}}$ are states. $\sigma_{c}(t):[0, \infty) \rightarrow\{1,2, \cdots, \rho\}$ is the switching signal. $A_{c s}(\omega)=A_{c s}$ $+\Delta A_{c s}, B_{c s}(\omega)=B_{c s}+\Delta B_{c s}, C_{c}(\omega)=C_{c}+\Delta C_{c}, S=1,2, \cdots, \rho . A_{c s}, B_{c S}$ and $C_{c}, D_{c}$ are constant matrices. Suppose that the switched linear system $x_{c}{ }_{c}=A_{c \sigma_{c}(t)} x_{c}$ is asymptotically stable under the switching signal $\sigma_{c}(t)$ and there exists an open neighborhood $\bar{\Omega}$ of $\omega=0$ and constant matrix $X_{c}(\omega)$ that satisfies the following linear matrix equations:

$$
\begin{gathered}
X_{c}(\omega) \Gamma=A_{c i}(\omega) X_{c}(\omega)+B_{c i}(\omega), \\
0=C_{c}(\omega) X_{c}+D_{c}, i=1,2, \cdots, \rho .
\end{gathered}
$$

Then, we have

$$
\lim _{t \rightarrow \infty} e(t)=0 .
$$

\section{Main results}

In this section, we give the solvability condition of robust output regulation problem of switched multi-agent systems.

We employ the merging technique in [31] to describe the switching signal of the switched multiagent sys- tems with $\sigma(t):[0, \infty) \rightarrow \prod_{1 \leq i \leq N} I_{i}$, where $\left.\sigma(t)=\left(\sigma_{1}(t)\right), \sigma_{2}(t), \cdots, \sigma_{N}(t)\right)$. The merging action is denoted by $\oplus$ such that $\sigma(t)=\sigma_{1}(t) \oplus \sigma_{2}(t) \oplus \cdots \oplus \sigma_{N}(t)$. This means that the switched multi-agent systems has $M_{1} \times M_{2} \times \cdots \times M_{N}$ and the set of switching times for $\sigma(t)$ is the union of the sets of switching times for $\sigma_{i}(t), i=1,2, \cdots, N$. Let the controller gain matrix $G_{i j_{i}}$ be as follows:

$$
G_{i j_{i}}=U_{i j_{i}}(\omega)-K_{i j_{i}} X_{i}(\omega), i=1,2, \cdots, N, j_{i} \in M_{i},
$$

where $X_{i}(\omega), U_{i j_{i}}(\omega)$ are the solutions of equations (6).

Let $A_{j}(\omega)=$ block diag $\left(A_{i j_{1}}(\omega), A_{2 j_{2}}(\omega), \cdots, A_{N_{j_{N}}}(\omega)\right), B_{j}(\omega)=\operatorname{block} \operatorname{diag}\left(B_{i j_{1}}(\omega), B_{2 j_{2}}(\omega), \cdots\right.$, $\left.B_{N j_{N}}(\omega)\right), E_{j}(\omega)=$ block diag $\left(E_{i j_{1}}(\omega), E_{2 j_{2}}(\omega), \cdots, E_{N j_{N}}(\omega)\right), K_{j}=\operatorname{block} \operatorname{diag}\left(K_{i j_{1}}, K_{2 j_{2}}, \cdots\right.$,

$\left.K_{N j_{N}}\right), G_{j}=$ block diag $\left(G_{i j_{1}}, G_{2 j_{2}}, \cdots, G_{N j_{N}}\right), C(\omega)=$ block $\operatorname{diag}\left(C_{1}(\omega), C_{2}(\omega), \cdots, C_{N}(\omega)\right), X(\omega)=$ block $\operatorname{diag}\left(X_{1}(\omega), X_{2}(\omega), \cdots, X_{N}(\omega)\right)$.

By (6) and (10), we have

$$
\begin{aligned}
X(\omega)\left(I_{N} \otimes S\right) & =\left(A_{j}(\omega)+B_{j}(\omega) K_{j}\right) X+\left(E_{j}(\omega)+B_{j}(\omega) G_{j}\right), \\
0 & =C(\omega) X(\omega)+\left(I_{N} \otimes F\right) .
\end{aligned}
$$

According to Assumption 3, there always exist $K_{i j_{i}}, i=1,2, \cdots, N, j_{i} \leq j_{i} \in M_{i}$, such that $A_{i j_{i}}+$ $B_{i j_{i}} K_{i j_{i}}$ are Hurwitz. Then, there always exist a family of constants $\lambda_{0 i}>0, \gamma_{0 i}>0, i=1,2, \cdots, N, j_{i}$ $\in M_{i}$ and positive definite matrices $P_{i j_{i}}$ such that

$$
\left(A_{i j_{i}}+B_{i j_{i}} K_{i j_{i}}\right)^{\mathrm{T}} P_{i j_{i}}+P_{i j_{i}}\left(A_{i j_{i}}+B_{i j_{i}} K_{i j_{i}}\right)+\gamma_{0 i} I+2 \lambda_{0 i} P_{i j_{i}}<0 .
$$


Definition 1[4]: For a switching signal $\sigma$ and any $T \geq t>0$, let $N_{\sigma}(t, T)$ be the switching numbers of $\sigma$ over the interval $(t, T)$. If for any given $N_{\sigma} \leq 0$ and $\tau_{a}>0$, we have $N_{\sigma}(t, T) \leq N_{0}+\frac{T-t}{\tau_{a}}$, then $\tau_{a}$ and $N_{0}$ are called average dwell time and the chatter bound, respectively.

We now have the solvability condition for the regulation problem.

Theorem 1: Let Assumptions 1-4 hold. Suppose that the inequalities (12) are established, the robust output regulation for the switched multi-agent systems (1) is solved by the controller (4) for any agent-dependent average dwell time

$$
\tau_{a i} \geq \tau_{a i}^{*}=\frac{\ln u_{i}}{2 \lambda_{i}}, \lambda \in\left(0, \lambda_{0 i}\right) .
$$

Proof: Under the distributed control law (5), the closed-loop system of $i$-th agent is

$$
\begin{aligned}
& \dot{x}_{i}=\left(A_{i j_{i}}(\omega)+B_{i j_{i}}(\omega) K_{i j_{i}}\right) x_{i}+B_{i j_{i}}(\omega) G_{i j_{i}} \xi_{i}+\mathrm{E}(\omega) v, \\
& \dot{\xi}=S \xi_{i}+\alpha\left[\sum_{j \in \Phi_{t}} a_{i j}\left(\xi_{j}-\xi_{i}\right)+a_{i 0}\left(\omega-\xi_{i}\right)\right] \\
& \dot{v}=S v \\
& e_{i}=C_{i}(\omega) x_{i}+F v .
\end{aligned}
$$

Let $x=\left(x_{1}^{T}, x_{2}^{T}, \cdots, x_{N}^{T}\right)^{T}, \xi=\left(\xi_{1}^{T}, \xi_{2}^{T}, \cdots, \xi_{N}\right)^{T}, \mathrm{e}=\left(e_{1}, e_{2}, \cdots, e_{N}\right)$ and $\tilde{v}=1_{N} \otimes v$. Thus, the overall closedloop system of the switched linear multi-agent system can be rewritten in the form.

$$
\begin{aligned}
& \dot{x}=\left(A_{\sigma(t)}(\omega)+B_{\sigma(t)}(\omega) K_{\sigma(t)}\right) x+B_{\sigma(t)}(\omega) G_{\sigma(t)} \xi+E_{\sigma(t)}(\omega) \tilde{v}, \\
& \xi=\left(\left(I_{N} \otimes S\right)-\alpha\left(H \otimes I_{q}\right)\right) \xi+\left(H \otimes I_{q}\right) \tilde{v}, \\
& \dot{\tilde{v}}=\left(I_{N} \otimes S\right) \tilde{v}, \\
& e=C(\omega) x+\left(I_{N} \otimes F\right) \tilde{v} .
\end{aligned}
$$

Next, we will prove the origin of the closed-loop system without the disturbance input .

$$
\begin{aligned}
& \dot{x}=\left(A_{\sigma(t)}(\omega)+B_{\sigma(t)}(\omega) K_{\sigma(t)}\right) x+B_{\sigma(t)}(\omega) G_{\sigma(t)} \xi, \\
& \dot{\xi}=\left(\left(I_{N} \otimes S\right)-\alpha\left(H \otimes I_{q}\right)\right) \xi .
\end{aligned}
$$

is asymptotically stable. Obviously, according to Assumption 1 and Assumption 4, $\left[\left(I_{N} \otimes S\right)-(H\right.$ $\left.\otimes I_{q}\right]$ ) is Hurwitz. Therefore, there exist a symmetric and positive-define matrix $Q$ and a positive constant $\gamma_{2}$ such that

$$
\left[\left(I_{N} \otimes S\right)-\alpha\left(H \otimes I_{q}\right)\right]^{\mathrm{T}} Q+Q\left[\left(I_{N} \otimes S\right)-\alpha\left(H \otimes I_{q}\right)\right]+\rho_{2} I<0
$$

For system (1), we define the following piecewise Lyapunov function candidate

$$
\begin{aligned}
V_{\sigma(t)}(x(t), \xi(t)) & =\Sigma^{N}{ }_{i=1} V_{i \sigma_{i}(t)}\left(x_{i}(t)\right)+k w(\xi(t)) \\
& =\Sigma^{N}{ }_{i=1} x_{i}(t)^{T} P_{i j_{i}} x_{i}(t)+k \xi(t)^{T} Q \xi(t),
\end{aligned}
$$

where positive constant $k$ will be given later. $\sigma(t)$ is the merged switching signal of the switching signals $\sigma_{i}(t), i=1,2, \cdots, N$ of all switched agents. This means that the set of switching times for $\sigma(t)$ is the union of the sets of switching times for $\sigma_{i}(t), i=1,2, \cdots, N$. According to this merged switching signal $\sigma(t), \sigma(t)=j$ means that for any $i$, the $j_{i}$-th subsystem of the $i$-th agent is activated. According to(12) and (18), we obtain

$$
\begin{gathered}
\dot{V}(x(t), \xi(t))=\sum_{i=1}^{N}\left(x_{i}(t)^{T} P_{i j i} x_{i}(t)+x_{i}(t)^{T} P_{i j i} x_{i}(t)\right)+k\left(\xi(t)^{\mathrm{T}} Q \xi(t)+\xi(t)^{\mathrm{T}} Q \xi(t)\right) \\
=\sum_{i=1}^{N} x_{i}^{T}(t)\left\{\left(A_{i j_{i}}+B_{i j_{i}} K_{i j_{i}}\right)^{\mathrm{T}} P_{i j_{i}}+P_{i j_{i}}\left(A_{i j_{i}}+B_{i j_{i}} K_{i j_{i}}\right) x_{i}(t)+2 x_{i}(t)^{\mathrm{T}} P_{i j_{i}} B_{i j_{i}} G_{i j_{i}} \xi_{i}(t)\right\}+k \xi^{\mathrm{T}}(t)\left\{\left[\left(I_{N}\right.\right.\right. \\
\left.\left.\quad \otimes S)-\alpha\left(H \otimes I_{q}\right)\right]^{\mathrm{T}} Q+Q\left[\left(I_{N} \otimes S\right)-\alpha\left(H \otimes I_{q}\right)\right]\right\} \xi(t) \\
\left.<\sum_{i=1}^{N}\left(-2 \lambda_{o i}\right) V_{i}\left(x_{i}(t)\right)-\lambda_{o i}\left\|x_{i}(t)\right\|^{2}-\gamma_{0 i}\left\|x_{i}(t)\right\|^{2}+2 x_{i}(t)^{\mathrm{T}} P_{i j i} B_{i j i} G_{i j i} \xi(t) i\right]-k \rho_{2}\|\xi(t)\|^{2} .
\end{gathered}
$$

We know that there exist constants $\alpha_{i}>0, \delta_{i}>0, i=1,2, \cdots, N$, such that 


$$
\left\|x_{i}^{T} P_{i j_{i}}\right\|^{2} \leq \alpha_{i}\left\|x_{i}(t)\right\|,\left\|B_{i j i} G_{i j i} \xi_{i}(t)\right\| \leq \delta_{i}\left\|\xi_{i}(t)\right\| .
$$

Let $\theta=\max \left\{\alpha_{i} \delta_{i}, i=1,2, \cdots, N\right\}$, we have

$$
\dot{V}(x(t), \xi(t)) \leq \sum_{i=1}^{N}\left(-2 \lambda_{o i}\right) V_{i}\left(x_{i}(t)\right)-\left(k \rho_{2}-\frac{\theta^{2}}{\lambda_{o i}}\right)\|\xi(t)\|^{2} .
$$

Since $P_{i \sigma_{i}(t)}, i=1,2, \cdots, N$ are switched among the solutions $P_{i j_{i}(t)}, i=1,2, \cdots, N, j_{i} \in M_{i}$ of (12) according to the piecewise constant switching signal $\sigma_{i}(t)$,there exist constants $\mu_{i} \geq 1$ such that

$$
\begin{gathered}
V_{i j_{i}}\left(x_{i}\right) \leq \mu_{i} V_{i j_{s}}\left(x_{i}\right), \quad i=1,2, \cdots, N, j_{i}, j_{s} \in M_{i} . \\
V(x(t), \xi(t)) \leq \sum_{i=1}^{N} \mu_{i}^{N_{i \sigma_{i}(0, t)}} e^{-2 \lambda_{0 i} t} V(x(0), \xi(0))-\left(k \rho_{2}-\frac{\theta}{\gamma_{0 i}}\right)^{2}\|\xi(t)\|^{2},
\end{gathered}
$$

where $\lambda_{i}=\max _{j_{i} \in M_{i}} \lambda_{i j_{i}}$. Under the average dwell time (13), we have

$$
V(x(t), \xi(t)) \leq \sum_{i=1}^{N} e^{-2\left(\lambda_{i}-\lambda_{o_{i}}\right) t} V(x(0), \xi(0) .
$$

Thus, the closed-loop system without disturbance input and $\omega=0$ is asymptotically stable for any agent-dependent average dwell time $\tau_{a i} \geq \tau_{a i}{ }^{*}$.

Next, Using lemma 2, we obtain $\lim _{t \rightarrow \infty} e(t)=0$. The proof is thus completed.

\section{Simulation example}

Consider the following switched multi-agent systems.

$$
\begin{aligned}
& x_{i}=A_{i j i}(\omega) x_{i}+B_{i j i}(\omega) u_{i}+E_{i j i}(\omega) v, \\
& y_{i}=C_{i}(\omega) x_{i}, \quad i=1,2,3 ; j_{1}=1,2 ; j_{2}=1,2 ; j_{3}=1,2 .
\end{aligned}
$$

where

$$
\begin{aligned}
& A_{11}=\left[\begin{array}{ll}
0 & 0 \\
0 & 1
\end{array}\right], A_{12}=\left[\begin{array}{cc}
-1 & 0 \\
1 & 2
\end{array}\right], B_{11}=\left[\begin{array}{l}
1 \\
0
\end{array}\right], B_{12}=\left[\begin{array}{l}
0 \\
1
\end{array}\right], E_{11}=\left[\begin{array}{cc}
-2 & 1 \\
0 & 1
\end{array}\right], E_{12}=\left[\begin{array}{cc}
-2 & -7 \\
1 & 2
\end{array}\right] ; A_{21}=\left[\begin{array}{ll}
1 & 1 \\
0 & 0
\end{array}\right], \\
& A_{22}=\left[\begin{array}{cc}
2 & 1 \\
0 & -3
\end{array}\right], B_{21}=\left[\begin{array}{l}
0 \\
1
\end{array}\right], B_{22}=\left[\begin{array}{l}
1 \\
0
\end{array}\right], E_{21}=\left[\begin{array}{cc}
3 & 0 \\
-1 & -2
\end{array}\right], E_{22}=\left[\begin{array}{cc}
2 & 1 \\
-12 & -8
\end{array}\right], A_{31}=\left[\begin{array}{ll}
0 & 0 \\
0 & 1
\end{array}\right], A_{32}=\left[\begin{array}{cc}
1 & 0 \\
0 & -2
\end{array}\right], \\
& B_{31}=\left[\begin{array}{l}
1 \\
0
\end{array}\right], B_{32}=\left[\begin{array}{l}
1 \\
0
\end{array}\right], E_{31}=\left[\begin{array}{cc}
1 & -3 \\
0 & -4
\end{array}\right], E_{32}=\left[\begin{array}{cc}
1 & -1 \\
-9 & -7
\end{array}\right], C_{1}=\left[\begin{array}{cc}
1 & 0 \\
-1 & 1
\end{array}\right], C_{2}=\left[\begin{array}{cc}
-1 & 1 \\
0 & -1
\end{array}\right] ; C_{3}=\left[\begin{array}{cc}
1 & 0 \\
0 & -1
\end{array}\right] \\
& F=\left[\begin{array}{cc}
1 & 2 \\
-3 & -1
\end{array}\right] ; S=\left[\begin{array}{ll}
1 & 1 \\
0 & 2
\end{array}\right]
\end{aligned}
$$

By Assumption 4, we give the matrix $H$ described the information exchange of the multi-agent systems as $H=\left[\begin{array}{cc}1 & -1 \\ 0 & 1\end{array}\right] . \Delta A_{i j_{i}}=0.01 * i * j_{i}, \Delta B_{i j_{i}}=0.005 * i * j_{i}, \Delta E_{i j_{i}}=0.02 * i * j_{i}, \Delta C_{i}=0.04 * i$.

We given the constant $\alpha=3$.Let $\gamma_{01}=0.5, \gamma_{02}=0.001, \gamma_{03}=0.2 ; \lambda_{1}=0.5, \lambda_{2}=2, \lambda_{3}=0.9 ; \tau_{a 1} \geq 2.14, \tau_{a 2} \geq$ $1.8, \tau_{a 3} \geq 10.5$. We obtain the simulation results as shown in Figs. 1 .

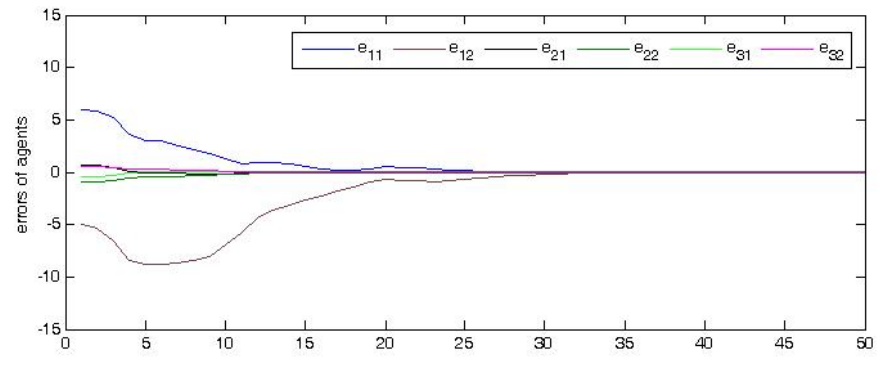

Fig. 1: errors of the three agents. 


\section{Conclusion}

In this paper, we have studied the robust output regulation problem of multi-agent systems whose each agent is a switched linear system. The switching dynamics of each agent bring us challenges for the research of the problem. Based on the classical average dwell time method, a new agentdependent average dwell time method is presented to solve the robust output regulation problem. Due to the limited information exchanges between agents, we establish a distributed controller on the basis of a distributed In the future, the study of the output regulation problem should be extend to multi-agent systems whose each agent is a nonlinear system.

\section{References}

[1] D. Liberzon, Switching in Systems and control, Boston, MA: Birkhauser, 2003.

[2] D. Liberzon, and A.S. Morse, Basic problems in stability and design of switched system, IEEE Control Syst. Mag.. 19(5) ( 1999) 59-70.

[3] A.S. Morse, Supervisory control of families of linear set-point controllers, part 1: Exact matching, IEEE Transactions on Automatic Control. 41 (1996) 1413-1431.

[4] J. Hespanha, and A.S. Morse, Stability of switched systems with average dwell-time, In Proceeding 38th IEEE Conf. on Decision and Control. 2655-2660, 1999.

[5] G.S. Zhai, B. Hu, K. Yasuda, and A. Michel, Stability Analysis of Switched Systems with Stable and Unstable Subsystems: An Average Dwell Time Approach, Proceedings of the American Control Conference, Chicago, Illinois June, 1677-1684, 2000.

[6] G. Lafferriere, A.Williams, J. Caughma, and J. Veerman, Decentralized control of vehicle formations, Systems and Control Letters. 54(9) (2005) 899-910.

[7] Y.F. Su, and J. Huang, Cooperative output regulation of linear multi-agent systems, Transaction on Automatic Control. 57(4) (2012) 1062-1066.

[8] Y.F. Su, and J. Huang, Cooperative output regulation of linear multi-agent systems by output feedback, Systems and Control Letters. 61 (2012) 1248-1253.

[9] Y.F. Su, Y.G. Hong, and J. Huang, A Result on the Cooperative Robust Output Regulation for Linear Uncertain Multi-Agent Systems, The 9th IEEE International Conference on Control and Automation (ICCA), Santiago, Chile, 19-21,2011.

[10] X.L. Wang, Y.G. Hong, J. Huang, and Z.P. Jiang, A Distributed Control Approach to A Robust Output Regulation Problem for Multi-Agent Linear Systems, IEEE Transaction Automatic Control. 55(12) (2010) 2891-2895.

[11] Y.F. Su, and J. Huang, Cooperative Output Regulation with Application to Multi- Agent Consensus Under Switching Network, IEEE Transactions on Systems, Man, and cybernetics part B: cybernetics, 42:864-875, 2012.

[12] Y.G. Hong, L.X. Gao, D.Z. Chen, and J.P. Hu, Lyapunov-Based Approach to Multi-agent Systems With Switching Jointly Connected Interconnection, IEEE Transactions on Automatic Control, 52 (2007) 943-948.

[13] X.L. Wang, W. Ni, and J. Yang, Distributed output regulation of switching multi-agent systems subject to input saturation, IET Control Theory and Applications. 7 (2013) 202-209.

[14] A. Cervantes-Herrera, J. Ruiz-Leon, C. Lopez-Limon, and A. Ramirez-Trevino, A distributed control design for the output regulation and output consensus of a class of switched linear multiagent systems, In Proceeding of IEEE 17th Conference on Emerging Technologies and Factory Automation, Krakow, Poland,1-7, 2012.

[15] C. Grodsil, and G. Royle, Algebraic Graph Theory, New York:Springer-Verlag, 2001.

[16] Y. Su, and J. Huang, Output Regulation of a Class of Switched Linear Multi-Agent Systems: a Distributed Observer Approach, In the 18 IFAC World Congress, Milano(Italy),2011. 